\title{
Tecnura
}

INVESTIGACIÓN

\section{Modelo AHP-VIKOR para handoff espectral en redes de radio cognitiva}

\section{AHP-VIKOR Model for Spectrum Handoff in Cognitive Radio Networks}

\author{
César Hernández*, Ingrid Páez**, Diego Giral***
}

Fecha de recepción: 30 de noviembre de 2014

Fecha de aceptación: 06 de abril de 2015

Como citar: Hernández, C., Páez, I., \& Giral, D. (2015). Modelo AHP-VIKOR para handoff espectral en redes de radio cognitiva. Revista Tecnura, 19(45), 29-39. doi:10.14483/udistrital.jour.tecnura.2015.3.a02

\section{RESUMEN}

En este artículo se presenta la propuesta de un algoritmo híbrido para la asignación de espectro en redes de radio cognitiva basado en los algoritmos Analytical Hierarchical Process (AHP) y Multi-Criteria Optimization and Compromise Solution (VIKOR), con el objetivo de mejorar el desempeño de la movilidad espectral de los usuarios secundarios en redes de radio cognitiva.

Para evaluar el nivel de desempeño del algoritmo propuesto se realiza un análisis comparativo entre este, el Grey Relational Analysis (GRA) y una asignación de espectro aleatoria (Random). Los dos primeros trabajan con los mismos criterios de decisión: probabilidad de disponibilidad del canal, tiempo estimado de disponibilidad del canal, relación señal a ruido más interferencia y ancho de banda. A diferencia de los trabajos relacionados, la evaluación comparativa se validó a través de una traza de datos reales de ocupación espectral capturados en la banda de frecuencia GSM, que modela el comportamiento real de los usuarios licenciados. En la evaluación de desempeño se utilizaron cinco métricas de evaluación: número promedio acumulado de handoff fallidos, número promedio acumulado de handoff realizados, ancho de banda promedio, retardo promedio acumulado y throughput promedio acumulado.

Los resultados del análisis comparativo con los otros dos algoritmos muestran que el algoritmo de handoff AHP-VIKOR propuesto provee el mejor desempeño en la movilidad espectral.

Palabras clave: handoff espectral, modelo híbrido, radio cognitiva, redes móviles.

\begin{abstract}
This paper proposed a hybrid algorithm for spectrum allocation in cognitive radio networks based on two algorithms, analytical hierarchical process (AHP) and multi-criteria optimization and compromise solution (VIKOR), for improving the performance of mobility spectrum of secondary users in cognitive radio networks.
\end{abstract}

* Ingeniero electrónico, magíster en ciencias de la información y las comunicaciones, candidato a doctor en Ingeniería de Sistemas y Computación de la Universidad Nacional de Colombia. Docente e investigador de la Universidad Distrital Francisco José de Caldas, Bogotá, Colombia. Contacto: cahernandezs@udistrital.edu.co

** Ingeniera eléctrica, ingeniera industrial y doctora en Ingeniería de Telecomunicaciones de la Universidad de Cantabria. Docente investigadora de la Universidad Nacional de Colombia, Bogotá, Colombia. Contacto: ippaezp@unal.edu.co

*** Ingeniero eléctrico, estudiante de la Maestría en Ingeniería Eléctrica, Universidad de los Andes, Bogotá, Colombia. Contacto: da.giral10@ uniandes.edu.co 
To evaluate the level of performance of the proposed algorithm, a comparative analysis between the proposed AHP-VIKOR, Grey Relational Analysis (GRA) and a random allocation of spectrum (Random) algorithm, is performed. The first two algorithms work with the same decision criteria: probability of channel availability, estimated time availability, signal-to-interference-plus-noise ratio and bandwidth. Unlike related work, benchmarking was validated through a trace of real spectral occupation data, captured in the GSM frequency band, which models the actual behavior of licensed users. For performance evaluation five metric were used, handoff failed average cumulative number, handoff average cumulative number, average bandwidth, delay and average cumulative throughput.

The results of the comparative analysis with the other two algorithms show that the AHP-VIKOR algorithm proposed provides the best performance in spectral mobility.

Keywords: spectral handoff, hybrid model, cognitive radio, mobile networks.

\section{INTRODUCCIÓN}

La creciente demanda de aplicaciones inalámbricas junto a las políticas de asignación fija del espectro radioeléctrico ha hecho que actualmente se reduzca la disponibilidad de bandas de frecuencia especialmente en aquellas con mayor demanda como las asignadas a las comunicaciones móviles. Sin embargo, investigaciones recientes han demostrado que a pesar de la escasez de bandas de frecuencia existe un uso poco eficiente del espectro radioeléctrico, con tiempos de utilización inferiores al 10\%, produciendo ciclos útiles prácticamente nulos (Forero, 2012). Dichos estudios concluyen que una de las mejores soluciones al problema del uso ineficiente del espectro radioeléctrico es la asignación dinámica del mismo.

La radio cognitiva es la tecnología capaz de realizar una asignación dinámica del espectro radioeléctrico. La radio cognitiva de acuerdo con la Unión Internacional de Telecomunicaciones (ITU) es "una radio o sistema que detecta y está al tanto de su entorno y se puede ajustar de forma dinámica y autónoma de acuerdo con sus parámetros de funcionamiento de radio". En la radio cognitiva a diferencia de la red tradicional existen dos tipos de usuarios, el usuario licenciado o primario (PU) que paga por utilizar una banda de frecuencia licenciada, y el usuario no licenciado o secundario (SU) que hace un uso oportunista del espectro licenciado del PU mientras esté disponible y liberar el recurso espectral cuando el PU lo requiera y buscar uno nuevo (I. F. Akyildiz, Lee Vuran \& Mohanty, 2008; I. F. Akyildiz, Lee Vuran, \& Mohanty, 2006).

El proceso mediante el cual el SU se cambia de un canal de frecuencia a otro se conoce como handoff espectral. Durante el handoff espectral el SU debe pausar su comunicación mientras realiza la detección del espectro, identifica las oportunidades espectrales (frecuencias disponibles), selecciona el canal objetivo y realiza el cambio de canal de frecuencia, para posteriormente continuar su comunicación (Ian F Akyildiz, Lee, \& Chowdhury, 2009).

Según la literatura actual (I. F. Akyildiz et al., 2008; Ian F. Akyildiz et al., 2006; Ian F Akyildiz et al., 2009; Christian, Moh, Chung, \& Lee, 2012; Marinho \& Monteiro, 2011; L.-C. Wang, Wang, \& Chang, 2012; Rodriguez-Colina, Ramirez P, \& Carrillo A, 2011; C.-W. Wang \& Wang, 2009), los esquemas de handoff espectral son determinantes para el desempeño de las comunicaciones del SU, a través de la reducción del número promedio de cambios de canal y el correspondiente nivel de latencia, garantizando una transmisión de datos continua en las comunicaciones del SU, por tanto, los modelos de handoff espectral son un tema importante en la radio cognitiva (Christian et al., 2012).

En la literatura actual se proponen varios esquemas de handoff espectral para redes inalámbricas 
cognitivas, sin embargo, el desempeño de cada uno es satisfactorio dentro del contexto para el cual fue diseñado.

Los modelos basados en toma de decisiones de múltiples criterios (MCDM) han sido utilizados en la radio cognitiva en los trabajos de investigación (Tsai, 2007; Yang \& Jung-ShyrWu, 2008; Zapata Cortés, Arango Serna, \& Adarme Jaimes, 2012; Yang Shin-Jer, 2013) para seleccionar el canal de frecuencia objetivo durante el handoff espectral. Aunque los problemas basados en MCDM son diversos, comparten características similares como: múltiples criterios de decisión y alternativas para seleccionar, así como un vector de pesos ponderados que representan la importancia relativa de cada criterio de decisión (E. Stevens-Navarro, Martínez-Morales, \& Pineda-Rico, 2013). Las características de los modelos MCDM, los convierten en una buena alternativa para modelar el proceso de handoff espectral. Algunos de los modelos de handoff espectral propuestos en el estado del arte son: Simple Additive Weighting (SAW) (Zhang, 2004), Technique for Order Preference by Similarity to Ideal Solution (TOPSIS) (Zhang, 2004), Multiplicative Exponent Weighting (MEW) (E. Stevens-Navarro \& Wong, 2006), Grey Relational Analysis (GRA) (Song \& Jamalipour, 2005), Elimination and Choice Translating Priority (ELECTRE) (Bari \& Leung, 2007), Weighted Markov Chain (WMC) (Ying, Jun, Yun, Gen, \& Ping, 2008) y Multicriteria Optimization and Compromise Solution (VIKOR) (Enrique Stevens-Navarro, Gallardo-Medina, Pineda-Rico, \& Acosta-Elias, 2012) y Analytical Hierarchical Process (AHP). El algoritmo AHP ha demostrado ser una alternativa eficaz para evaluar y seleccionar las mejores oportunidades espectrales (E. Stevens-Navarro, Lin, \& Wong, 2008; Mohamed, Leghris, \& Adib, 2011; Song \& Jamalipour, 2005; Kibria, Jamalipour, \& Mirchandani, 2005).

Este trabajo propone un modelo de handoff espectral basado en un algoritmo híbrido que combina las ventajas de la técnica Analytical Hierarchical Process (AHP) y Multi-Criteria Optimization and Compromise Solution (VIKOR). Este algoritmo permite mejorar la toma de decisiones a la hora de seleccionar dinámicamente una oportunidad espectral con base en los siguientes criterios de decisión: probabilidad de disponibilidad (PD) del canal, tiempo estimado de disponibilidad (TED) del canal, SINR y ancho de banda (BW). A través de la técnica AHP se determina el peso de cada uno de los criterios de decisión y con VIKOR se realizar un ranking de cada una de las potenciales oportunidades espectrales.

Para evaluar el nivel de desempeño del modelo de handoff propuesto se realiza un análisis comparativo con otros dos modelos: Grey Relational Analysis (GRA) y una asignación de espectro aleatoria (Random). VIKOR-AHP y GRA trabajan con los mismos cuatro criterios de decisión. A diferencia de los trabajos relacionados, la evaluación comparativa se validó a través de una traza de datos reales de ocupación espectral capturados en la banda de frecuencia de la tecnología Global System for Mobile Communications (GSM), lo cual permitió incluir el comportamiento real de los PU dentro de las simulaciones realizadas.

El resto del artículo está estructurado como sigue. En la sección II se describen los tres modelos de handoff considerados. En la sección III se presentan los resultados alcanzados en el análisis comparativo de la evaluación de desempeño de los modelos de handoff espectral y, finalmente, en la sección IV se presentan las conclusiones.

\section{METODOLOGÍA}

En esta sección se presenta el diseño de los tres modelos de handoff espectral por comparar para la toma de decisiones en redes de radio cognitiva: algoritmo AHP-VIKOR, algoritmo GRA y el algoritmo aleatorio.

\section{Algoritmo AHP-VIKOR}

Como se mencionó anteriormente, este algoritmo híbrido combina las ventajas de AHP y VIKOR. Primero determina los pesos de los cuatro criterios 
de decisión a través de AHP y después realiza un ranking de las oportunidades espectrales por medio de VIKOR.

El método AHP es un algoritmo de estimación para la toma de decisiones multicriterio, que permite incluir varios criterios tanto cuantitativos como cualitativos. Lo anterior hace que el algoritmo AHP pueda evaluar diversas variables al momento de seleccionar una única opción dentro de un amplio conjunto de alternativas posibles, basándose en comparaciones de la importancia entre criterios escogidos para la selección de una alternativa, siendo esta más una medida relativa que un valor absoluto (Saaty, 1990).

A continuación se describen los cinco pasos realizados en el desarrollo del algoritmo AHP.

1) Definición del problema: En la metodología de diseño del algoritmo AHP, el primer paso es definir el problema, descomponiéndolo a su vez en objetivo, criterios y alternativas. El objetivo es la decisión que se ha de tomar, que para el presente trabajo es muy clara y corresponde a la selección de la mejor oportunidad espectral disponible.

Los criterios son los factores que afectan la preferencia de una alternativa. Para este caso se seleccionaron cuatro criterios: PD, TED, SINR y AB.

Las alternativas son todas las opciones que existen y de las cuales hay que escoger solo una. En este caso, corresponden a los canales de frecuencia que se encuentran disponibles al momento de realizar la ejecución del modelo de handoff espectral.

2) Construcción de la jerarquía: Con los criterios seleccionados, junto al objetivo y las alternativas del algoritmo de decisión multicriterio AHP, se construye la jerarquía.

3) Matrices de juicios: Una vez construida la jerarquía se procedió a realizar las matrices de juicios, las cuales corresponden a evaluaciones comparativas que definen el nivel de importancia relativa entre cada combinación posible de parejas de criterios.

4) Cálculo de los pesos normalizados: Con las matrices de juicios definidas, se procedió a calcular los pesos normalizados para cada criterio con base en el modelo propuesto por Eduardo Miranda (2001) debido a su sencillez y la calidad de sus resultados, fundamentado en la media geométrica.

El primer paso es calcular la media geométrica $V_{i}$ de cada fila de la matriz de juicios definida para un determinado criterio, el cual está definido por la ecuación (1).

$$
v_{i}=\sqrt[n]{\prod_{j=1}^{n}} a_{i j}
$$

Donde,

$V_{i}$ es la media geométrica de la fila i,

n es el número de subcriterios,

j representa las columnas de la matriz de juicios, $y$

$a_{i j}$ es el valor del elemento de la fila i y columna j.

El segundo paso es calcular el vector de valores propios $r$, el cual define los pesos normalizados de cada criterio y que está definido por la ecuación (2).

$$
r=\left[r_{1}, r_{2}, \ldots ., r_{n}\right] \text { con } r_{i}=\frac{v_{i}}{\sum_{j=1}^{n} v_{j}}
$$

Donde,

$r$ es el vector de valores propios,

$r_{1}, r_{2}, \ldots, r_{n}$ es el valor de los pesos de cada subcriterio,

$V_{i}$ es la media geométrica de la fila i, y

$V_{j}$ es la media geométrica de la columna j.

5) Cálculo del índice de consistencia: Con el objetivo de evaluar la validez del algoritmo AHP se calculó el índice de consistencia. De acuerdo con Saaty (1990), si el índice de consistencia es menor que 0.1, el desarrollo del algoritmo es satisfactorio. Para calcular el índice de consistencia se utilizó la ecuación (3) (Miranda, 2001).

${ }^{C} C I=\frac{\sqrt{\sum_{i=1}^{n} \sum_{j=1}^{n}\left(\ln a_{i j} \sum \ln \frac{v_{i}}{v_{j}}\right)^{2}}}{\frac{(n \Sigma 1) \times(n \Sigma 2)}{2}}$

$\mathrm{Cl}$ es el índice de consistencia,

n el número de subcriterios,

i el valor de la fila de la matriz de juicios, 
j el valor de la columna de la matriz de juicios, $a_{i j}$ es el valor del elemento de la fila i y columna $\mathrm{j}$, $V_{i}$ es la media geométrica de la fila i, y

$V_{j}$ es la media geométrica de la columna j.

El método VIKOR asume que cada alternativa es evaluada de acuerdo con cada función de criterio, y la clasificación puede ser desarrollada a través de la comparación de las medidas que estén más cercanas a la alternativa ideal (Tanino, Tanaka, \& Inuiguchi, 2003). El algoritmo VIKOR sigue los siguientes pasos (E. Stevens-Navarro et al., 2013):

Para cada parámetro $j=1,2,3, \ldots, N$, se determina el mejor y peor valor dado por la ecuación (4) y (5).

$$
\begin{aligned}
& F_{j}^{+}=\left\{\left(\max _{i \in M} x_{i j} \mid j \in N_{b}\right),\left(\min _{i \in M} x_{i j} \mid j \in N_{c}\right)\right\}, \\
& F_{j}^{-}=\left\{\left(\min _{i \in M} x_{i j} \mid j \in N_{b}\right),\left(\max _{i \in M} x_{i j} \mid j \in N_{c}\right)\right\},
\end{aligned}
$$

Donde $N_{b}$ que pertenece a $\mathrm{N}$ es el conjunto de parámetros de beneficios y $N_{c}$ que pertenece a $\mathrm{N}$ es el conjunto de parámetros de costos.

Se calcula los valores de $S_{i}$ y $R_{i}$ para $i=$ $1,2,3, \ldots, M$, dado por la ecuación (6) y (7).

$$
\begin{gathered}
S_{i}=\sum_{j \in N} w_{j} \frac{\left(F_{j}^{+}-x_{i j}\right)}{\left(F_{j}^{+}-F_{j}^{-}\right)^{\prime}} \\
R_{i}=\max _{i \in N}\left[w_{j} \frac{\left(F_{j}^{+}-x_{i j}\right)}{\left(F_{j}^{+}-F_{j}^{-}\right)}\right],
\end{gathered}
$$

Donde $W_{j}$ es la importancia del peso del parámetro j.

Se calcula los valores de $Q_{i}$ para $\mathrm{i}=1,2,3, \ldots$, M, dado por la ecuación (8).

$$
\begin{aligned}
& Q_{i}=\gamma\left(\frac{S_{i}-S^{+}}{S^{-}-S^{+}}\right)+(1-\gamma)\left(\frac{R_{i}-R^{+}}{R^{-}-R^{+}}\right) \\
& \text {Donde: } S^{+}=\min _{i \in M} S_{i}, \quad S^{-}=\max _{i \in M} S_{i}, \\
& R^{+}=\min _{i \in M} R_{i}, \quad R^{-}=\max _{i \in M} R_{i} \text { y } 0 \leq \gamma \leq 1
\end{aligned}
$$

pertenece al peso de la estrategia.

Dados los valores para Q, para todos los i pertenecientes a $M$, se clasifican de mayor a menor las oportunidades espectrales candidatas. Finalmente, la oportunidad espectral seleccionada está dada por la ecuación (9).

$$
\mathrm{A}_{\mathrm{VIK}}^{*}=\arg \min _{\mathrm{i} \in \mathrm{M}} \mathrm{Q}_{\mathrm{i}}^{*}
$$

\section{Algoritmo GRA}

El objetivo de este algoritmo es establecer las redes candidatas y seleccionar las que tengan más alta puntuación de acuerdo con unos parámetros definidos. Para lograr esto se establecen relaciones de Grey entre elementos de dos series: la primera contiene las mejores cualidades, mientras que la otra contiene entidades comparativas. Acá es parte importante el coeficiente de Grey, que se usa para describir las relaciones entre las series calculado a partir del nivel de similitud y variabilidad (Ramírez Pérez \& Ramos Ramos, 2010). El método GRA tiene los siguientes pasos (Ramirez-Perez \& Ramos, 2012)it is crucial to integrate heterogeneous networks so as to provide collective services. Vertical handoff consists in handing off the traffic flows of applications running on a mobile device through heterogeneous networks in order to keep or improve the QoS. In this work, we propose an algorithm for decision making in vertical handoff which considers three active applications running on a mobile device. The selectivity of our algorithm can be adjusted as a function of a set of QoS requirements e.g., the minimal bit rate, delay, jitter, and packet error rate (PER:

Primero se generar el vector de referencia $X_{o}$ de la matriz $X$, a través de la escogencia de los valores mínimos para los costos y los valores máximos para los criterios de beneficios.

Después, la secuencia de datos debe ser normalizada para $X$ de acuerdo con tres situaciones: más grande el mejor, más pequeño el mejor o nominal el mejor.

Luego, se calcula el coeficiente relacional de Grey como lo describe la ecuación (10).

$$
\gamma\left(x_{0}(i), x_{j}(i)\right)=\frac{\Delta_{\min }+\zeta \Delta_{\max }}{\Delta_{0, j}(i)+\zeta \Delta_{\max }}
$$


Donde,

$\Delta_{0, j}=\left|x_{0}(i)-x_{j}(i)\right|$

$\Delta_{\min }=\min _{j \in N}\left\{\min _{i \in M}\left\{\left|x_{0}(i)-x_{j}(i)\right|\right\}\right\}$

$\Delta_{\max }=\max _{j \in N}\left\{\max _{i \in M}\left\{\left|x_{0}(i)-x_{j}(i)\right|\right\}\right\}$

Donde el coeficiente $\zeta$ que pertenece $[0,1]$, compensa el efecto de $\Delta_{\max }$ que generalmente es 0,5 .

Finalmente, se calcula el grado relacional de Grey para cada una de las diferentes series de datos. $\Gamma\left(x_{0}, x_{i}\right)$ representa el grado relacional de Grey para las j-ésimas alternativas, (ecuación (11)).

$$
\Gamma\left(x_{0}, x_{j}\right)=\sum_{i=1}^{M} \omega_{i} \gamma\left(x_{0}(i), x_{j}(i)\right)
$$

Donde el peso de la importancia para los i-ésimos criterios es $W_{i}$.

\section{Algoritmo Aleatorio}

En el caso del algoritmo aleatorio, se desarrolló una selección totalmente aleatoria dentro de un conjunto de alternativas compuesto por todas las oportunidades espectrales potenciales.

\section{RESULTADOS}

La evaluación del desempeño de los tres modelos de handoff espectral se fundamentó en los resultados obtenidos a través de cinco métricas de evaluación: el número acumulado de handoff faIlidos, el número acumulado de handoff totales, el promedio de ancho de banda, el retardo promedio acumulado y el throughput promedio acumulado. Los resultados alcanzados en esta investigación del análisis comparativo para cada una de las métricas mencionadas se pueden observar en las figuras 1 , 2, 3, 4 y 5. La tabla 1 resume el desempeño de cada uno de los tres modelos de handoff espectral.

La figura 1 describe el número acumulado de handoff fallidos que se presentaron en cada modelo de handoff espectral durante una transmisión de 10 minutos, realizada a partir de datos de ocupación espectral reales. De la figura 1 también se observa que el algoritmo AHP-VIKOR propuesto tiene un comportamiento casi lineal, con un número muy bajo de handoff fallidos en comparación con los algoritmos GRA y Random. Por el contrario, el algoritmo Random tiene el comportamiento mas irregular, con el mayor número de handoff fallidos, aproximadamente 30 veces mas que el algoritmo AHP-VIKOR.

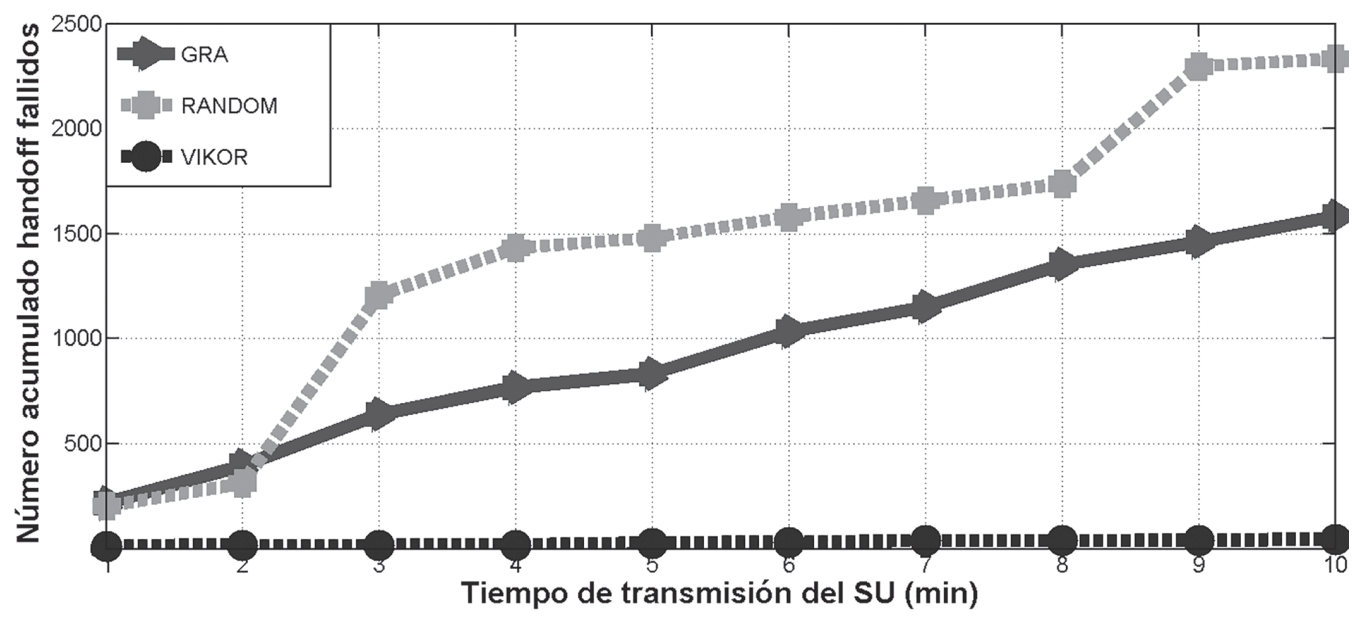

Figura 1. Número acumulado de handoff fallidos.

Fuente: elaboración propia. 
La figura 2 describe el número acumulado de handoff totales que se presentaron en cada modelo de handoff espectral durante una transmisión de 10 minutos, realizada a partir de datos de ocupación espectral reales.

La figura 3 describe el promedio de ancho de banda que se presentó en cada modelo de handoff espectral durante una transmisión de 10 minutos, realizada a partir de datos de ocupación espectral reales. De la figura 3 también se observa que aunque el algoritmo GRA describe un comportamiento menos variable que los otros dos algoritmos, su nivel de ancho de banda es relativamente muy bajo. Los algoritmos AHP-VIKOR y Random a pesar de presentar mayor variación en el ancho de banda, tienen un nivel promedio ocho y siete veces más alto que el algoritmo GRA, respectivamente.

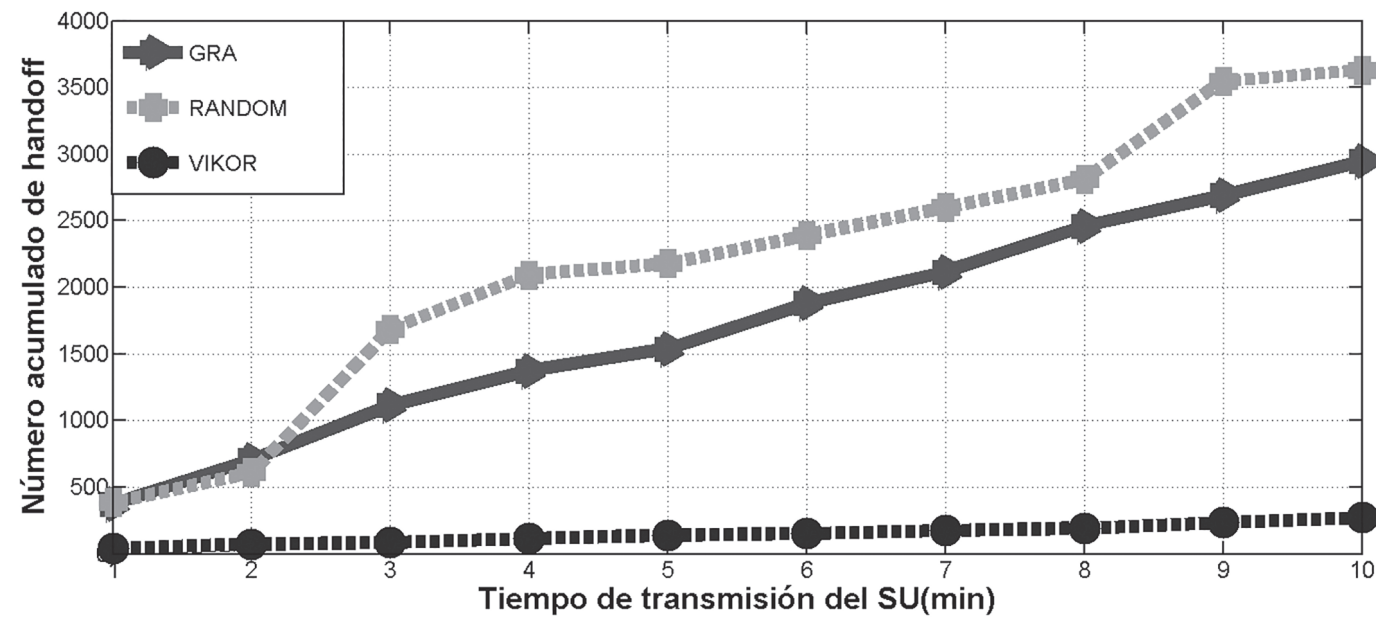

Figura 2. Número acumulado de handoff totales.

Fuente: elaboración propia.

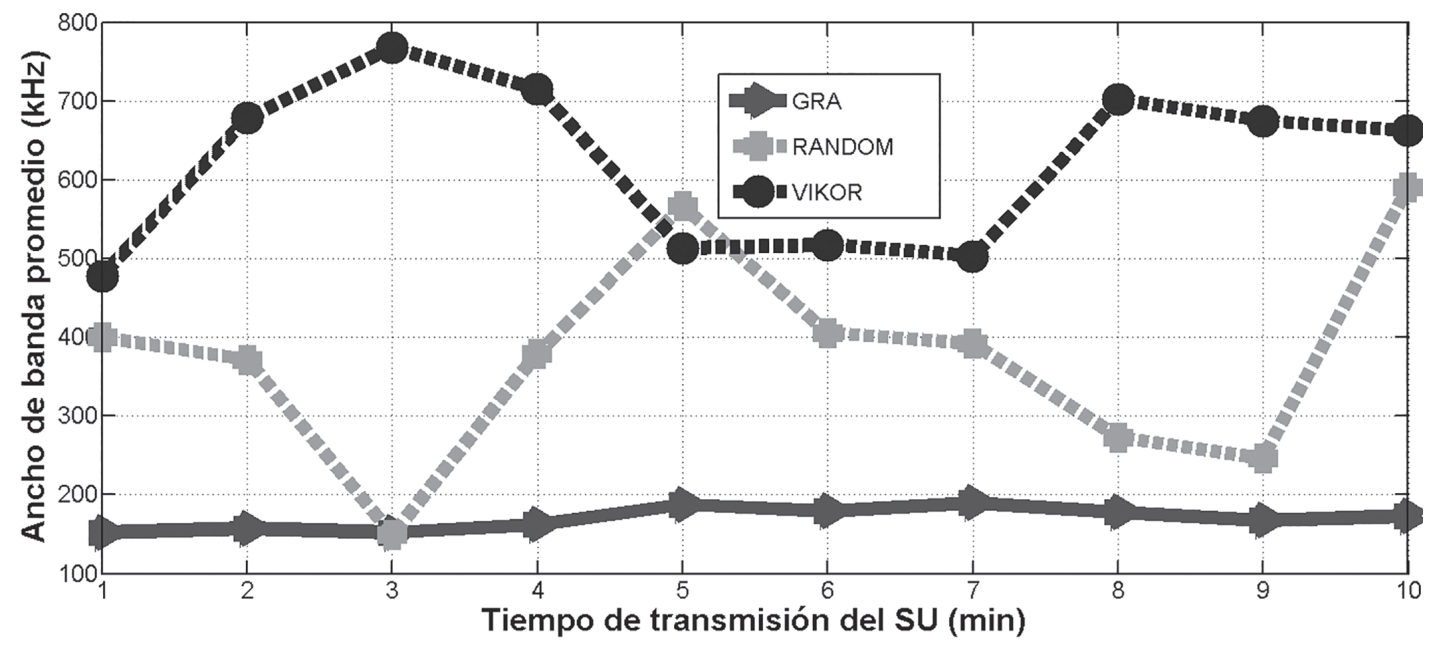

Figura 3. Ancho de banda promedio.

Fuente: elaboración propia. 
La figura 4 describe el retardo promedio que se presentó en cada modelo de handoff espectral durante una transmisión de 10 minutos, realizada a partir de datos de ocupación espectral reales. Para determinar los retardos promedios acumulados de cada algoritmo, se tuvo en cuenta el número de handoff fallidos y exitosos (corresponden a la diferencia entre los handoff totales y los fallidos). La figura 4 describe un buen comportamiento del algoritmo AHP-VIKOR propuesto en comparación con los algoritmos GRA y Random, los cuales muestran un comportamiento exponencial.

La figura 5 describe el throughput promedio que se presentó en cada modelo de handoff espectral durante una transmisión de 10 minutos, realizada a partir de datos de ocupación espectral reales.

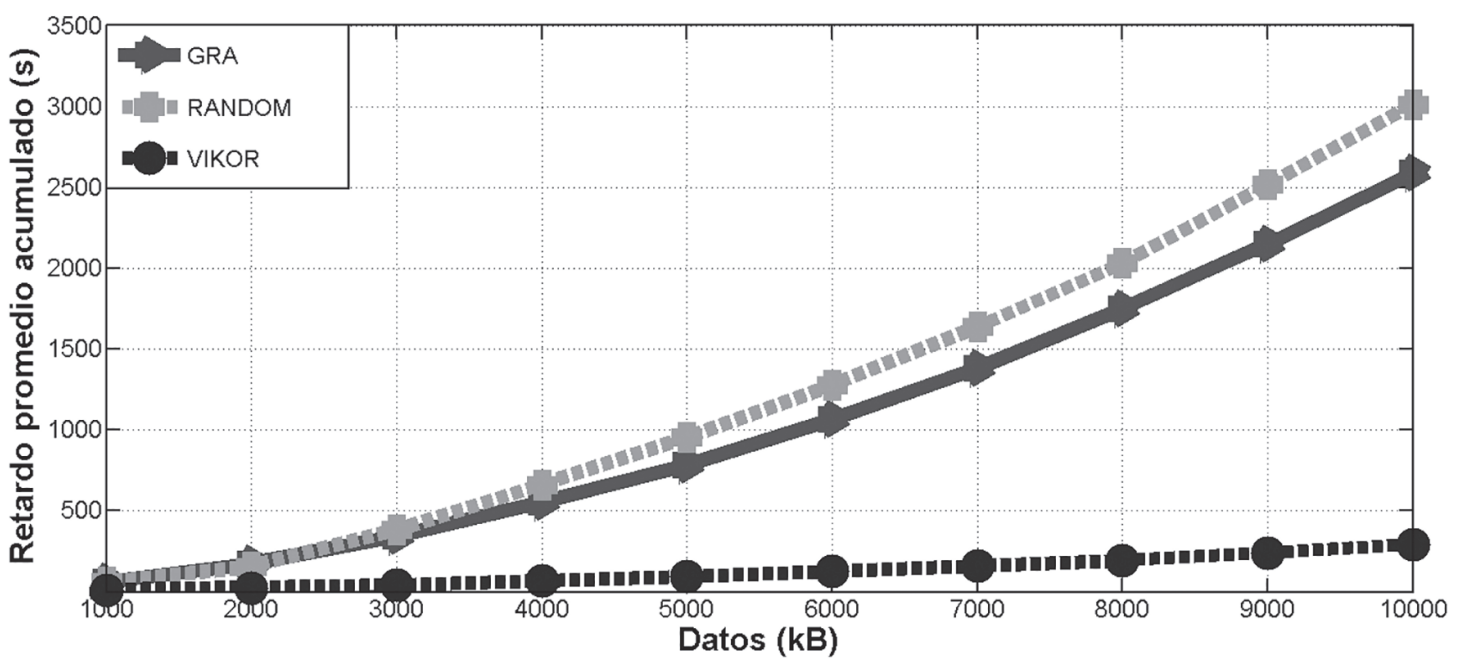

Figura 4. Retardo promedio.

Fuente: elaboración propia.

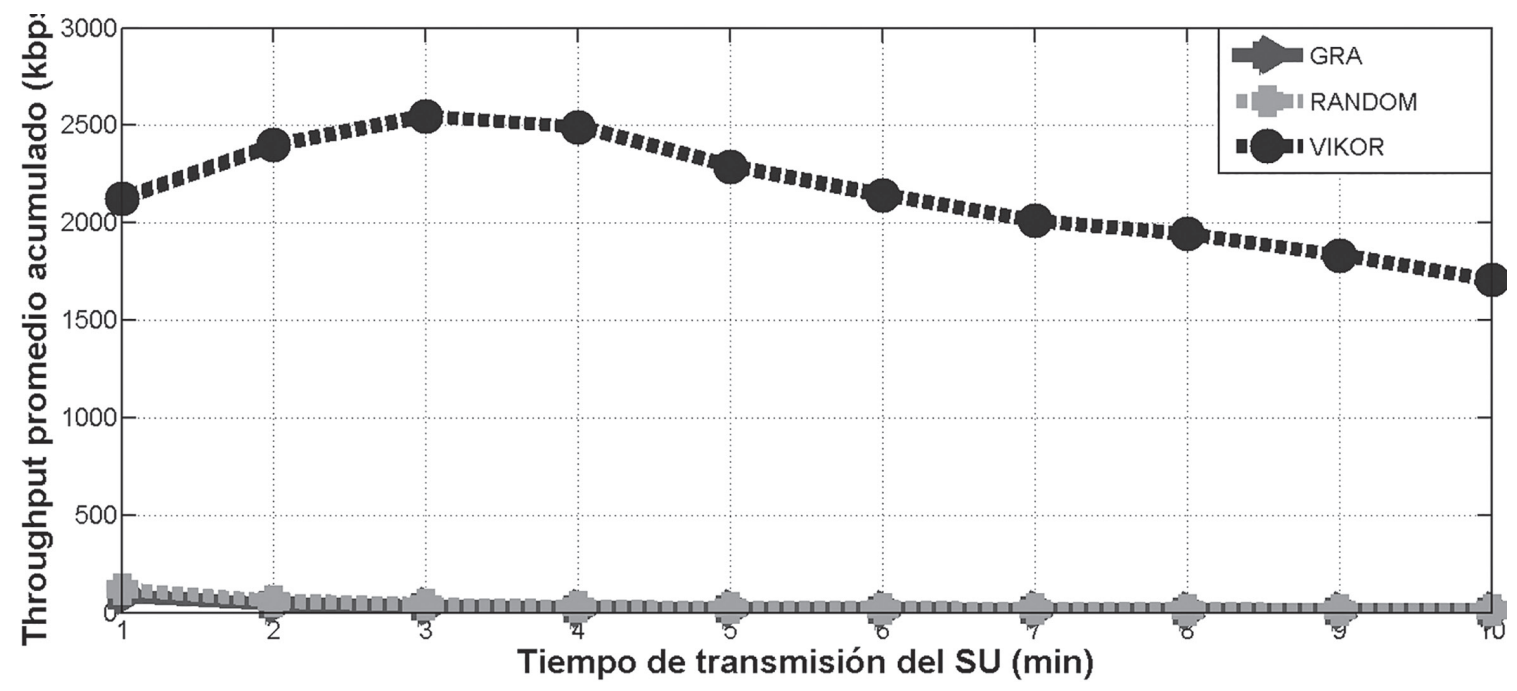

Figura 5. Throughput promedio.

Fuente: elaboración propia. 
Tabla 1. Evaluación comparativa de los tres modelos de handoff espectral

\begin{tabular}{lcccccc}
\hline \multirow{2}{*}{ Algoritmo } & \multicolumn{6}{c}{ Evaluación comparativa } \\
\cline { 2 - 7 } & $\begin{array}{c}\text { Handoff } \\
\text { fallidos }\end{array}$ & $\begin{array}{c}\text { Handoff } \\
\text { totales }\end{array}$ & $\begin{array}{c}\text { Ancho } \\
\text { de banda }\end{array}$ & Retardo & Throughput & $\begin{array}{c}\text { Desempeño } \\
\text { final }\end{array}$ \\
\hline AHP-VIKOR & Bajo & Bajo & Alto & Bajo & Alto & Alto \\
\hline GRA & Alto & Alto & Bajo & Alto & Muy Bajo & Bajo \\
\hline RANDOM & Muy Alto & Muy Alto & Medio & Muy Alto & Muy Bajo & Muy Bajo \\
\hline
\end{tabular}

Fuente: elaboración propia.

Para determinar el throughput promedio acumulado de cada algoritmo, se utilizó como estándar la modulación 16QAM. La figura 5 describe un comportamiento del algoritmo AHP-VIKOR muy superior en comparación con los algoritmos GRA y Random.

La tabla 1 describe la evaluación comparativa de los tres modelos de handoff espectral para redes de radio cognitiva, en términos de las cinco métricas de evaluación ejecutadas.

\section{CONCLUSIÓN}

De acuerdo con los resultados obtenidos a través de las simulaciones realizadas, a partir de los datos de ocupación espectral reales, el algoritmo híbrido para toma de decisiones multicriterio AHP-VIKOR es una técnica relativamente sencilla, que proporciona un eficiente y eficaz proceso de selección de canales de frecuencia. Lo anterior permite mejorar y fortalecer el desempeño de las estrategias de handoff espectral en las redes de radio cognitiva, en especial las proactivas.

La heterogeneidad de las redes inalámbricas actuales imponen retos en la práctica de las redes de radio cognitiva debido a las diferencias en la infraestructura de las redes actuales. Lo anterior refuerza la importancia de contar con modelos de handoff espectral que puedan adaptarse a las características de dichas redes y obtener el mejor desempeño posible para las comunicaciones de los usuarios secundarios sin afectar las comunicaciones de los usuarios primarios. Cada modelo de handoff espectral es útil para el contexto para el que fue diseño, por tal razón un multi-modelo podría ser una solución interesante.

\section{FINANCIAMIENTO}

Colciencias y el Centro de Investigaciones y Desarrollo Científico de la Universidad Distrital Francisco José de Caldas

\section{REFERENCIAS}

Akyildiz, I. F., Lee, W.-Y., \& Chowdhury, K. R. (2009). CRAHNs: Cognitive Radio Ad Hoc Networks. Ad Hoc Netw., 7(5), 810-836. http://doi.org/10.1016/j. adhoc.2009.01.001

Akyildiz, I. F., Lee, W.-Y., Vuran, M. C., \& Mohanty, S. (2006). NeXt generation/dynamic spectrum access/ cognitive radio wireless networks: A survey. Computer Networks, 50(13), 2127-2159. http://doi.org/10.1016/j.comnet.2006.05.001

Akyildiz, I. F., Lee, W.-Y., Vuran, M. C., \& Mohanty, S. (2008). A survey on spectrum management in cognitive radio networks. IEEE Communications Magazine, 46(4), 40-48. http://doi.org/10.1109/ MCOM.2008.4481339

Bari, F., \& Leung, V. (2007). Application of ELECTRE to Network Selection in A Hetereogeneous Wireless Network Environment (pp. 3810-3815). http://doi. org/10.1109/WCNC.2007.697

Christian, I., Moh, S., Chung, I., \& Lee, J. (2012). Spectrum mobility in cognitive radio networks. IEEE Communications Magazine, 50(6), 114-121. http:// doi.org/10.1109/MCOM.2012.6211495 
Forero, F. (2012, November). Detección de códigos de usuarios primarios para redes de radio cognitiva en un canal de acceso DCMA. Colombia.

Kibria, M. R., Jamalipour, A., \& Mirchandani, V. (2005). A location aware three-step vertical handoff scheme for 4G/B3G networks (Vol. 5, pp. 2752-2756). http://doi.org/10.1109/GLOCOM.2005.1578260

Marinho, J., \& Monteiro, E. (2011). Cognitive radio: survey on communication protocols, spectrum decision issues, and future research directions. Wireless Networks, 18(2), 147-164. http://doi.org/10.1007/ s11276-011-0392-1

Miranda, E. (2001). Improving subjective estimates using paired comparisons. IEEE Software, 18(1), 87-91. http://doi.org/10.1109/52.903173

Mohamed, L., Leghris, C., \& Adib, A. (2011). A Hybrid Approach for Network Selection in Heterogeneous Multi-Access Environments (pp. 1-5). http://doi. org/10.1109/NTMS.2011.5720658

Ramírez Pérez, C., \& Ramos Ramos, V. M. (2010). Handover vertical: un problema de toma de decisión múltiple. In VIII Congreso Internacional sobre Innovación y Desarrollo Tecnológico. Cuernavaca Morelos, México. Retrieved from http://mcyti.izt.uam. mx/ARTICULOS/ramirezperezCIINDET2010.pdf

Ramirez-Perez, C., \& Ramos, R. V. M. (2012). A QoS hierarchical decision scheme for vertical handoff (pp. 1-4). http://doi.org/10.1109/ ICCDCS.2012.6188942

Rodriguez-Colina, E., Ramirez P, C., \& Carrillo A, C. E. (2011). Multiple attribute dynamic spectrum decision making for cognitive radio networks (pp. 1-5). http://doi.org/10.1109/WOCN.2011.5872960

Saaty, T. L. (1990). How to make a decision: The analytic hierarchy process. European Journal of Operational Research, 48(1), 9-26. http://doi. org/10.1016/0377-2217(90)90057-I

Song, Q., \& Jamalipour, A. (2005). A network selection mechanism for next generation networks (Vol. 2, pp. 1418-1422 Vol. 2). http://doi.org/10.1109/ ICC.2005.1494578

Stevens-Navarro, E., Gallardo-Medina, R., Pineda-Rico, U., \& Acosta-Elias, J. (2012). Application of MADM method VIKOR for vertical handoff in heterogeneous wireless networks. IEICE Transactions on Communications, 95(2), 599-602.

Stevens-Navarro, E., Lin, Y., \& Wong, V. W. S. (2008). An MDP-Based Vertical Handoff Decision Algorithm for Heterogeneous Wireless Networks. IEEE Transactions on Vehicular Technology, 57(2), 12431254. http://doi.org/10.1109/TVT.2007.907072

Stevens-Navarro, E., Martinez-Morales, J. D., \& Pineda-Rico, U. (2013). Evaluation of Vertical Handoff Decision Algorightms Based on MADM Methods for Heterogeneous Wireless Networks. Journal of Applied Research and Technology, 10(4), 534538. Retrieved from http://www.revistas.unam.mx/ index.php/jart/article/view/40602

Stevens-Navarro, E., \& Wong, V. W. S. (2006). Comparison between Vertical Handoff Decision Algorithms for Heterogeneous Wireless Networks (Vol. 2, pp. 947-951). http://doi.org/10.1109/ VETECS.2006.1682964

Tanino, T., Tanaka, T., \& Inuiguchi, M. (2003). Multi-Objective Programming and Goal Programming. Berlin, Heidelberg: Springer Berlin Heidelberg.

Tsai, M.-C. (2007). Multi-Attributes Handover Decision Mechanism across Wi-Fi \& WiMAX Using MIH Services.

Wang, C.-W., \& Wang, L.-C. (2009). Modeling and Analysis for Proactive-Decision Spectrum Handoff in Cognitive Radio Networks. In IEEE International Conference on Communications, 2009. ICC '09 (pp. 1-6). http://doi.org/10.1109/ICC.2009.5199189

Wang, L.-C., Wang, C.-W., \& Chang, C.-J. (2012). Modeling and Analysis for Spectrum Handoffs in Cognitive Radio Networks. IEEE Transactions on Mobile Computing, 11(9), 1499-1513. http://doi. org/10.1109/TMC.2011.155

Yang, S.-F., \& Jung-ShyrWu. (2008). A IEEE 802.21 Handover design with QOS provision across WLAN and WMAN (pp. 548-552). http://doi.org/10.1109/ ICCCAS.2008.4657833

Yang Shin-Jer, T. W.-C. (2013). Design novel weighted rating of multiple attributes scheme to enhance handoff efficiency in heterogeneous wireless networks. Computer Communications, 36(14), 1498-1514. http://doi.org/10.1016/j.comcom.2013.06.005 
Ying, W., Jun, Y., Yun, Z., Gen, L., \& Ping, Z. (2008). Vertical Handover Decision in an Enhanced Media Independent Handover Framework (pp. 2693-2698). http://doi.org/10.1109/WCNC.2008.472

Zapata Cortés, J. A., Arango Serna, M. D., \& Adarme Jaimes, W. (2012). Applying fuzzy extended analytical hierarchy (FEAHP) for selecting logistics software. Ingeniería E Investigación, 32(1), 94-99. Retrieved from http://www.revistas.unal.edu.co/index.php/ ingeinv/article/view/28521/33581

Zhang, W. (2004). Handover decision using fuzzy MADM in heterogeneous networks (Vol. 2, pp. 653-658 Vol.2). http://doi.org/10.1109/ WCNC.2004.1311263

\section{(c) $9 \underset{\mathrm{BY}}{\mathrm{N}} \mathrm{O}$}


